\title{
Capacidades para la innovación tecnológica: Un análisis en los hoteles de categoría superior en Puerto Vallarta, México
}

José Luis Bravo Silva

j.luis.bravo@gmail.com

Universidad de Guadalajara, Guadalajara, Jalisco, México.

\section{Manuel Ernesto Becerra Bizarron} mabebi9@hotmail.com

Universidad de Guadalajara, Guadalajara, Jalisco, México.

\section{Elba Martina Cortes Palacios}

cortes elba@hotmail.com Jalisco, México.

\section{RESUMEN}

La presente investigación analiza la innovación tecnológica realizada en los hoteles de categoría superior en Puerto Vallarta, Jalisco, México a partir de una encuesta aplicada a una muestra de gerentes operativos involucrados. La hotelería representa una actividad clave en el sector turístico mexicano, los hoteles de categoría superior captan el $45 \%$ de los visitantes con hospedaje en Puerto Vallarta, es ahí donde radica la importancia económica del sector y su vanguardia tecnológica. Los avances tecnológicos acelerados han incrementado sustancialmente la productividad y han permitido incrementar la oferta en mayor proporción de la demanda, en la actualidad se cuenta con una amplia gama de satisfactores para cubrir una misma necesidad. La capacidad de adopción tecnológica en una empresa hotelera repercute en la mejora de eficiencia y reducción de costos. El análisis de la adopción tecnológica, permite reflejar el potencial competitivo del sector en el largo plazo. La investigación describe a través del modelo de capacidades de innovación de Cohen y Levinthal, el conocimiento previo relacionado, la capacidad de aprendizaje, la capacidad de absorción y su traducción en la capacidad de innovación tecnológica de los gerentes operativos de los hoteles.
\end{abstract}

PALABRAS-CLAVE: Capacidades, Innovación tecnológica, hoteles. 


\section{INTRODUCCIÓN}

De acuerdo al Índice de Competitividad en Viajes y Turismo 2015, México se encuentra en la posición número 44 en competitividad turística a nivel mundial. En la región de América, se ubica el lugar 5. Estas posiciones denotan una importante labor por realizar por parte de la industria turística en conjunto a fin de poder incrementar la competitividad. La actividad turística representa a nivel global, una fuente de empleo directo para 120 millones de personas y 125 millones de empleos indirectos por las industrias relacionadas al turismo, lo que representa uno de cada 11 empleos en el mundo World Economic Forum (2015). La clave para el futuro de cualquier país o institución reside en el talento, habilidades y capacidades de sus habitantes. La empresa hotelera debe descubrir que capacidades debe desarrollar para incrementar la mezcla de talento humano con la que cuenta.

En términos de innovación, México se ubica en la posición 63 en el Índice Global de Innovación del WEF con importantes retos por atender. De acuerdo a la última medición realizada por Instituto Nacional de Estadística Geografía e Informática (INEGI, 2012) la innovación e investigación, en los servicios de hospedaje en conjunto con el sector de preparación de hospedaje a nivel nacional, solamente $10 \%$ de los establecimientos grandes, considerados como tales aquellos con un número de empleados superior a 50 , disponen de un departamento dedicado total o parcialmente al diseño o creación de nuevos productos o procesos, el $12 \%$ invierten en la creación de nuevos productos, materiales, dispositivos o componentes y el $1.60 \%$ registra productos u otras obras de creación intelectual ante institutos de propiedad intelectual, el $29.80 \%$ capacita al personal en el uso de nuevas tecnologías o procesos de trabajo, el $28.25 \%$ implementa procesos de reorganización en los sistemas de trabajo y el $41.57 \%$ adaptan sus bienes o servicios a los cambios en las preferencias de sus clientes. Solamente el $5 \%$ emplea equipo de cómputo en procesos administrativos y $3 \%$ emplea internet en sus relaciones con clientes y proveedores, $2 \%$ emplea equipo de cómputo en procesos técnicos o de diseño, $2 \%$ desarrolla programas o paquetes informáticos para mejorar sus procesos, lo que habla de la incipiente innovación en el sector, en mayor medida en la innovación de carácter tecnológico.

El cuarto lugar nacional, según las cifras del último censo económico disponible, lo ocupa el estado de Jalisco en donde se ubica Puerto Vallarta, el destino turístico bajo análisis. Jalisco, aporta al Producto Interno Bruto nacional $6.3 \%$ del total. El sector de actividad económica con mayor aportación al Producto Interno Bruto es el sector comercio, en donde los servicios de restaurantes y hoteles aportan el $25.1 \%$. Dada la relevancia económica de la hotelería para el país y para el estado, resulta importante mantener y acrecentar la aportación de este sector. Puerto Vallarta ocupó la tercera posición a nivel estado en cuanto a número de visitantes, representando un incremento en ocupación hotelera de $1.17 \%$ con respecto al año anterior, totalizando ocupación anual de $62.73 \%$ la tendencia en la afluencia de turistas y derrama económica por turista en Puerto Vallarta en los últimos 6 años ha sido creciente Secretaría de Turismo del Estado de Jalisco (SETUJAL).El gasto que realiza el turista en el destino se aplica primordialmente en el hospedaje en $36.98 \%$, lo cual implica que el hospedaje es, dentro de las actividades turísticas, la más relevante en términos de generación de ingresos para el destino. Estas tendencias sugieren encontrar 
alternativas en la gestión de los establecimientos de hospedaje del destino que busquen contribuir a elevar la competitividad.

La tecnología es una herramienta que facilita y energiza a las economías basadas en el conocimiento. La omnipresencia creciente del internet ha implicado que las actividades personales y de negocios se conduzcan de manera incremental en línea, por lo cual existe una emergencia de capacidades para capturar, almacenar y guardar estas interacciones. El crecimiento explosivo de las redes móviles, la computación en la nube y tecnologías de información y comunicación inteligentes, facilitan amplios campos de información que pueden ser procesados, compartidos y transferidos a través del mundo. Estos datos representan materia prima para la innovación, facilitando significativamente la creación de valor, beneficios sociales y ganancias en productividad(TORRENT, 2009; SCHLEICHER, 2011). Los desarrollos en tecnología de información han cambiado radicalmente la forma en la que las empresas de hospedaje operan, aunque la industria no está orientada a la tecnología por naturaleza, las demandas crecientes de consumidores sofisticados obliga al sector hotelero a adaptar tecnologías en sus procesos a fin de ser competitivos (LAW; LEUNG; AU; LEE, 2012).

La capacidad de adopción tecnológica en una empresa hotelera puede representar mejora de eficiencia y reducción de costos. Analizar la adopción tecnológica, permite identificar el potencial competitivo del sector en el largo plazo. La investigación describe a través del modelo de capacidades de innovación de Cohen y Levinthal (1990), el conocimiento previo relacionado, la capacidad de aprendizaje, la capacidad de absorción y su traducción en la capacidad de innovación tecnológica de los gerentes operativos de los hoteles.

\section{REVISIÓN TEÓRICA}

\section{LA INNOVACIÓN Y SUS CARACTERÍSTICAS}

El proceso de innovación es un flujo continuo de generación de conocimiento mediante diferentes procesos de aprendizaje (estudio, práctica, uso y error). El aprendizaje por práctica y por uso tiene un carácter incremental, el conocimiento creado se suma al conocimiento pre existente de la empresa generando recombinaciones y nuevos conocimientos. Por tanto es un proceso continuo (NIETO, 2003).

Las innovaciones radicales son manifestaciones de una acumulación de pequeños cambios previos. Rosenberg (1994) identifica que las empresas dedican $80 \%$ de su esfuerzo innovador a la mejora, innovaciones incrementales y el $20 \%$ al desarrollo de productos completamente nuevos o innovaciones radicales. La propensión a la innovación incremental es mayor que la radical.

Nieto (2003) identifica el carácter continuo de la innovación con conceptos extendidos en el ámbito de la dirección de empresas: "la mejora continua (IMAI, 1989), los árboles o racimos tecnológicos (GEST, 1986), la espiral de creación de conocimiento (NONAKA, 1995), la dirección estratégica basada en desarrollo de capacidades esenciales (PRAHALAD \& HAMEL, 1990), entre otros, se sustentan en modelos donde implícitamente subyace el supuesto de continuidad. 
La capacidad de innovación de una empresa está determinada por su trayectoria pasada Cohen y Levinthal (1990) de modo que las decisiones de hoy, van a condicionar la innovación en el futuro. Por tanto la innovación presenta un carácter acumulativo. Este hecho se aprecia claramente al analizar las estructuras cognitivas del individuo que forma las organizaciones y provee los conocimientos y experiencia para la innovación.

La experiencia de desarrollar una tarea de aprendizaje influye y mejora el desarrollo de aprendizajes futuros. Esta mejora progresiva hace que individuos aprendan a aprender. Lo mismo sucede con la capacidad de resolver problemas: los métodos que se emplean para resolver un problema constituyen el conocimiento previo relacionado que permite al individuo adquirir capacidades relativas a la resolución de problemas futuros. La posesión de conocimiento y destrezas relevantes favorece la creatividad, permitiendo distintos tipos de asociaciones y vínculos que nunca antes habían sido considerados (COHEN; LEVINTHAL, 1990). El aprendizaje por experiencia, el aprendizaje por desbordamiento de experiencia acumulada en un sector y el aprendizaje conjunto en la red de negocios, resultaron relevantes para mejoras y reducciones de costos (TANG; POPP, 2016). La disponibilidad, habilidad y posibilidad para innovar combinadas son componentes para la innovación organizacional y la renovación sistemática resultaron relevantes para la innovación en hoteles(BINDER; MAIR; STUMMER; KESSLER, 2016). La compatibilidad, el tamaño de establecimiento, la competencia tecnológica y la masa critica que cada hotel atiende fueron factores relevantes en la adopción tecnológica en hoteles. (WANG; LI; LI; ZHANG, 2016). En otros estudios, la experiencia para gestionar la inovacion se identifica como un factor clave en las organizaciones (MARTINEZROS ; ORFILA-SINTES, 2012).

Derivado de la revisión teórica, se identifica que el aprendizaje del individuo en el pasado resulta crucial para aprender con mayor rapidez y menor costo. En la actualidad marcará la dirección para procesos de innovación más asequibles y menos costosos en el futuro. En este sentido, la innovación efectuada en el pasado marcara la capacidad de innovación actual.

\section{MODELO DE CAPACIDAD DE ABSORCIÓN}

Cohen y Levinthal (1990) definen la capacidad de absorción como la habilidad para reconocer el valor de nueva información externa, asimilarla y aplicarla con fines comerciales. La capacidad de innovación de un individuo depende de su conocimiento previo relacionado. A medida que el conocimiento previo aumenta, se mejoran otras dos capacidades, la capacidad de adquisición de conocimiento, la habilidad de poner nuevo conocimiento en la memoria y la capacidad de recordar y utilizar los conocimientos instalados en ella, lo que contribuye según algunos psicólogos a reforzar el aprendizaje debido a que la memoria se desarrolla a partir de la asociación de ideas previas y nuevas, tal y como se refiere en la figura 1. 


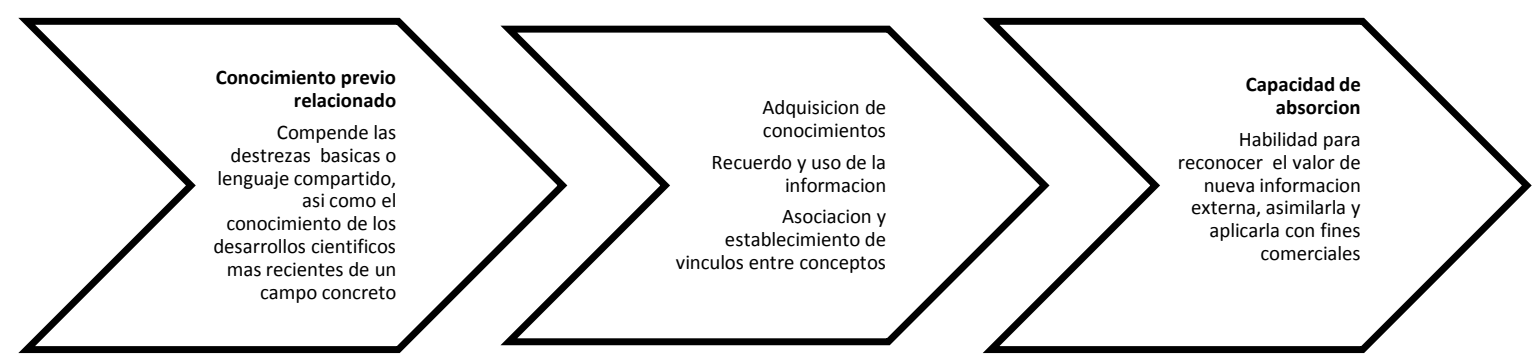

Fuente: Cohen y Levinthal (1990)

Cohen y Levinthal (1990) señalan por tanto que la capacidad de innovación se construye a partir del aprendizaje pasado, la resolución de problemas pasados que constituye el conocimiento previo relacionado que influye en la capacidad de absorción. Capacidades de absorción débiles, limitan el potencial de las capacidades de innovación de las empresas (MARTINEZ, GARCÍA; SANTOS, 2013). La capacidad de aprendizaje y la capacidad para resolver problemas generan la capacidad de innovación de una organización y en consecuencia que la empresa innove. La adopción tecnológica en la industria de la hospitalidad se esta ampliando (LAW; BUHALIS; COBANOGLU, 2014).

\section{METODOLOGÍA}

El diseño de la investigación es de tipo no experimental, transeccional, correlacional. Se observaron y midieron situaciones existentes en su contexto. Se correlacionaron los datos propuestos por el modelo de Cohen y Levinthal para efecto de relacionar la experiencia previa y la capacidad de innovación con la innovación tecnológica en los hoteles. Además sedescribieron las relaciones a través de una correlación de las variables.

El universo fueron los hoteles de Gran Turismo y Categoría Especial de Puerto Vallarta, debido a que se considera que por características de tamaño y competitividad, la variable de innovación tecnológica es altamente posible en este tipo de establecimientos de los cuales se eligieron los elementos muestrales de manera aleatoria, que fueron los directivos de las empresas, resultando una muestra de 68 directivos. Para la recolección de información se diseñó un instrumento de recolección de 16 reactivos con escalas de medición tipo Likert, mismo que fue validado con un Alpha de Chronbach de 0.98 para medir la consistencia en las respuestas. A través de las escalas sumadas se obtuvieron las 
puntuaciones por variable, mismas que fueron correlacionadas para emitir los resultados. Las variables consideradas fueron: nivel de estudios, experiencia en hotelería y antigüedad en el hotel, como referentes de conocimiento previo relacionado, del modelo de Cohen y Levinthal (1990). La capacidad de innovación fue medida para este estudio a través de la adaptación al cambio de los directivos, el nivel propositivo de cambios y la anticipación de cambios en la labor desempeñada, la generación de ideas fueron descriptivas de la capacidad de innovación, las cuales fueron relacionadas con el nivel de innovación tecnológica, descrito por los gerentes.

\section{RESULTADOS}

La tabla 1 describe los resultados obtenidos en la correlación de las variables. En el aspecto de grado de estudios del personal empleado como parte del conocimiento previo que haría proclive a la innovación, el resultado de la correlación con la innovación tecnológica, resulto de 0.082 que es de baja magnitud y no significativa. Lo que implica en el caso particular de estudio existe relación de las variables en sentido positivo, pero de baja magnitud.

La experiencia en hotelería de los gerentes, presenta relación con lainnovación tecnológica positiva con valor de 0.26 , ambas varían en el mismo sentido, a mayor experiencia en hotelería, mayor la tendencia a la innovación tecnológica.

Con respecto a la antigüedad del empleado en el hotel, señala que la relación es inversa mas no es significativa. Esto indica pese a no contar con significancia, que entre menor sea la antigüedad del gerente más proclive será a proponer innovaciones tecnológicas dada la relación negativa.

El constructo de capacidad de innovación de los empleados tiene una relación positiva, significativa y de alta magnitud, representada por el valor de 0.637

La capacidad de generación de ideas y la implementación de las mismas está relacionada con la innovación tecnológica de manera significativa y con una fuerte magnitud de 0.711 la mayor de todas las variables relacionadas en este estudio. Esto se puede observar en la tabla 1. 
Tabla 1 - Correlación de Pearson de variables de estudio

\begin{tabular}{|c|c|c|c|c|c|c|c|}
\hline & & $\begin{array}{c}\text { Grado } \\
\text { de } \\
\text { estudios }\end{array}$ & $\begin{array}{l}\text { Experiencia } \\
\text { en hotelería }\end{array}$ & $\begin{array}{l}\text { Antigüedad } \\
\text { en el hotel }\end{array}$ & $\begin{array}{l}\text { Capacidadde } \\
\text { innovación }\end{array}$ & $\begin{array}{c}\text { Generación } \\
\text { de ideas }\end{array}$ & $\begin{array}{l}\text { Innovación } \\
\text { Tecnológica }\end{array}$ \\
\hline $\begin{array}{l}\text { Grado de } \\
\text { estudios }\end{array}$ & $\begin{array}{c}\text { Correlación } \\
\text { de Pearson } \\
\text { Sig. } \\
\text { (bilateral) }\end{array}$ & 1 & $\begin{array}{r}-.130 \\
.291\end{array}$ & $\begin{array}{l}-.049 \\
.692\end{array}$ & $\begin{array}{l}.079 \\
.524\end{array}$ & $\begin{array}{l}.181 \\
.140\end{array}$ & $\begin{array}{l}.082 \\
.508\end{array}$ \\
\hline Experiencia & $\begin{array}{c}\text { Correlación } \\
\text { de Pearson } \\
\text { Sig. } \\
\text { (bilateral) }\end{array}$ & $\begin{array}{r}-.130 \\
.291\end{array}$ & 1 & $\begin{array}{l}.228 \\
.061\end{array}$ & $\begin{array}{l}.274^{*} \\
.024\end{array}$ & $\begin{array}{l}.170 \\
.166\end{array}$ & $\begin{array}{l}.265^{*} \\
.029\end{array}$ \\
\hline Antigüedad & $\begin{array}{c}\text { Correlación } \\
\text { de Pearson } \\
\text { Sig. } \\
\text { (bilateral) }\end{array}$ & $\begin{array}{l}-.049 \\
.692\end{array}$ & $\begin{array}{l}.228 \\
.061\end{array}$ & 1 & $\begin{array}{l}-.041 \\
.737\end{array}$ & $\begin{array}{c}-.258^{*} \\
.033\end{array}$ & $\begin{array}{l}-.201 \\
.100\end{array}$ \\
\hline $\begin{array}{l}\text { Capacidad } \\
\text { de } \\
\text { innovación }\end{array}$ & $\begin{array}{c}\text { Correlación } \\
\text { de Pearson } \\
\text { Sig. } \\
\text { (bilateral) }\end{array}$ & $\begin{array}{l}.079 \\
.524\end{array}$ & $\begin{array}{l}.274^{*} \\
.024\end{array}$ & $\begin{array}{l}-.041 \\
.737\end{array}$ & 1 & $\begin{array}{l}.803^{* *} \\
.000\end{array}$ & $\begin{array}{l}.637^{* *} \\
.000\end{array}$ \\
\hline $\begin{array}{l}\text { Generación } \\
\text { de ideas }\end{array}$ & $\begin{array}{c}\text { Correlación } \\
\text { de Pearson } \\
\text { Sig. } \\
\text { (bilateral) }\end{array}$ & $\begin{array}{l}.181 \\
.140\end{array}$ & $\begin{array}{l}.170 \\
.166\end{array}$ & $\begin{array}{l}-.258^{*} \\
.033\end{array}$ & $\begin{array}{l}.803^{* *} \\
.000\end{array}$ & 1 & $\begin{array}{l}.711^{* *} \\
.000\end{array}$ \\
\hline $\begin{array}{l}\text { Innovación } \\
\text { Tecnológica }\end{array}$ & $\begin{array}{c}\text { Correlación } \\
\text { de Pearson } \\
\text { Sig. } \\
\text { (bilateral) }\end{array}$ & $\begin{array}{l}.082 \\
.508\end{array}$ & $.265^{*}$ & $\begin{array}{l}-.201 \\
.100\end{array}$ & $\begin{array}{l}.637^{* *} \\
.000\end{array}$ & $\begin{array}{l}.711^{* *} \\
.000\end{array}$ & 1 \\
\hline
\end{tabular}

*. La correlación es significativa en el nivel 0,05 ( 2 colas).

**. La correlación es significativa en el nivel 0,01 ( 2 colas).

Fuente: Elaboración propia

\section{CONSIDERACIONES FINALES}

El estudio arroja resultados que podrían ser de utilidad para la gestión del capital humano en la hotelería de Puerto Vallarta. De acuerdo a los resultados obtenidos, la generación de ideas y la capacidad de innovación, que dentro del modelo de Cohen y Levinthal, los hace proclives a la innovación tecnológica. Proponer innovaciones tecnológicas depende de acuerdo a las correlaciones 
obtenidas en mayor medida de los conocimientos posteriores que de la experiencia previa.

Aunque el grado de estudios y la experiencia en hotelería que forman parte del conocimiento previo del empleado, se relacionan con la capacidad de proponer innovaciones tecnológicas, un empleado nuevo será más proclive a proponer innovaciones que un empleado con mayor antigüedad, de acuerdo a los resultados de este estudio. La verdadera tarea en una organización hotelera para lograr vanguardia tecnológica, dependerá más de la capacidad de innovación y generación de ideas, hecho que coincide con los hallazgos de la gestion de la inovacion se identifica como un factor clave junto con la experiencia en las organizaciones(MARTINEZ-ROS; ORFILA-SINTES, 2012).

Los entornos facilitadores en la organización para la propuesta de innovaciones tecnológicas, la promoción de una cultura de innovación y la recompensa por nuevas ideas, podrían tener efecto en la vanguardia tecnológica de un hotel.

Las limitantes de esta investigación radican en el hecho de tratarse de un caso particular, para un contexto específico y que el estudio no considera otras variables externas que también podrían influir en la adopción tecnológica de una empresa. 


\title{
Capacity for technological innovation: An analysis of higher category hotels in Puerto Vallarta, Mexico
}

\begin{abstract}
The aim of this paper is to analize how technological innovation is carried out in superior category hotels in Puerto Vallarta, México based on the results of a survey applied to operative managers of hotels. Hospitality represents a key economic activity in México. In Puerto Vallarta $45 \%$ of visitors stay at a superior category hotel. Technological advantage is key to be competitive. Increased technological advances representincreases in productivity in order to outperform offerings in higer rates tan demand. Nowadays competitive scenario for hotels is unclear, adoption capabilities based on technology becames a major factor to suceed. Technological innovation will reflect in cost reduction and an increase in efficency. This analysis is key to identify future competitive gaps in the sector.This paper describes based on Cohen and Levinthal innovation capabilities model, how previous knowledge, absorptive capability and technological innovation is being carried out among operative hotel managers.
\end{abstract}

KEYWORDS: Capabilities, Technological Innovation, hotels. 


\title{
Capacidade para inovação tecnológica: Uma análise nos hotéis de categoria superior em Puerto Vallarta, México
}

\begin{abstract}
RESUMO
Esta pesquisa analisa a inovação tecnológica feita em superior hotéis em Puerto Vallarta, Jalisco, México a partir de uma pesquisa com uma amostra de gerentes operacionais envolvidos. A indústria hoteleira é uma atividade fundamental do setor do turismo mexicano, superior hotéis capturar $45 \%$ dos visitantes com hospedagem em Puerto Vallarta, este é o lugar onde reside a importância econômica do setor e na vanguarda tecnológica. Acelerados avanços tecnológicos aumentaram substancialmente a produtividade e oaumentoda oferta tem permitido uma maior proporção de demanda, agora ele tem uma ampla gama de satisfações para cobrir a mesma necessidade. A capacidade da adoção da tecnologia em uma empresa hoteleira afeta a melhoria da eficiência e redução de custos. A análise da adoção da tecnologia, pode refletir o potencial competitivo do setor a longo prazo. A pesquisa descrita através de recursos de modelo de inovação Cohen e Levinthal, o conhecimento prévio relacionado, a capacidade de aprendizagem, capacidade de absorção e sua tradução para a capacidade de inovação tecnológica dos gerentes operacionais destes hotéis.
\end{abstract}

PALAVRAS-CHAVE: Capacidade. Inovação tecnológica. Hotéis. 


\section{REFERENCIAS}

CHESBROUGH, H. W. Open Innovation: the new imperative for creating and profiting from technology. Boston: Harvard Business School Publishing Corporation. 2003.

COHEN, W. M.;LEVINTHAL, D. A. Absorptive Capacity: A New Perspective on Learning and Innovation. Administrative Science Quarterly.1990.

EUROPEAN COMISSION. Innovation management and the knowledge-driven economy. brussels-luxembourg. 2004.

FERNANDEZ SANCHEZ, E.Innovacion, tecnologia y alianzas estrategicas. madrid: civitas. 1996.

FORREST, J. E. Model of the process of technological innovation. 439-453. 1991.

Guerra, D. Metodología para Dinamizar los Sistemas de Innovación. México: IPN. 2005.

HIDALGO NUCHERA, A.;LEON SERRANO, G.;PAVON MOROTE, J. la gestion de la inovacion y la tecnologia en las organizaciones. madrid: piramide. 2002.

KLINE, S.;ROSENBERG, N. An overview of innovation, the positive sum strategy. Washington D.C. 1986.

LAW, R.;BUHALIs, D.;COBANOGLU, C. Progress on information and communication technologies in hospitality and tourism. International Journal of Contemporary Hospitality Management, 727-750. 2014.

MARTINEZ, A.;GARCÍA, A.;SANTOS, G. Aprendizaje tecnológico en la industria manufacturera de Guanajuato. Frontera Norte, 187-212. 2013.

MARTINEZ-ROS, E.;ORFILA-SINTES, F. Training plans, manager's characteristics and innovation in the accommodation industry. International Journal of Hospitality Management, 686-694. 2012.

MÉXICO COMPETITIVO. Índice de Complejidad Económica. Obtenido de 
OECD Eurostat. Oslo Manual:Guidelines for collecting and interpreting innovation data. Obtenido de $<$ http://epp.eurostat.ec.europa.eu/cache/ITY PUBLIC/OSLO/EN/OSLO-EN.PDF>. 2005.

PARRA, E.; CALERO, F. Gestion y direccion de empresas turisticas. Madrid: Mc Graw Hill. 2006.

ROTHWELL, R. Towards the fifth-generation innovation process. En international marketing review vol.11 (págs. 7-31), 1994.

TANG, T.;POPP, D. (2016). The Learning Process and Technological Change in Wind Power: Evidence from China's CDM Wind Projects. Journal of Policy Analysis and Management, 195.

VELASCO, E. Z. (10 de MARZO de 2008). Universidad de La Rioja. Obtenido de $<$ http://dialnet.unirioja.es/servlet/fichero_articulo?codigo=2499438\&orden=0>. 2008 .

WANG, Y.-S.;LI, H.-T.;LI, C.-R.;ZHANg, D.-Z. Factors affecting hotels' adoption of mobile reservation systems: $A$ technology-organization-environment framework. Tourism Management, 163-172. 2016.

WORLD ECONOMIC FORUM. (2013). The Human Capital Report. Obtenido de <http://www3.weforum.org/docs/WEF HumanCapitalReport 2013.pdf>.

WORLD ECONOMIC FORUM. (2014). The Global Competitiveness Report 20142015: Full Data Edition. Obtenido de < $<$ ttp://reports.weforum.org/globalcompetitiveness-report-2014-2015/economies/\#economy=MEX>. 2014. 
Recebido: 07 fev. 2016.

Aprovado: 03 abr. 2016

DOI: $10.3895 /$ rbpd.v5n1.4419

Como citar: SILVA, J. L. B.; BIZARRON, E. B.; PALACIOS, E. M. C. Capacity for technological innovation:

an analysis of higher category hotels in Puerto Vallarta, Mexico. R. bras. Planej. Desenv., Curitiba, v. 5, n. 1,

p. 80-92, jan./abr. 2016. Disponível em: <https://periodicos.utfpr.edu.br/rbpd>. Acesso em: XXX.

Correspondência:

José Luis Bravo Silva

Av. Juárez, 976, Centro, 44100 Guadalajara, Jalisco, México

Direito autoral: Este artigo está licenciado sob os termos da Licença CreativeCommons-Atribuição 4.0

Internacional.

$$
\text { (c) (1) }
$$

